\title{
Platelet function monitoring with the Sonoclot analyzer after in vitro tirofiban and heparin administration
}

\author{
Michael A. Tucci, MD, DEAA, ${ }^{\text {a* }}$ Michael T. Ganter, MD, DEAA, ${ }^{\text {b* }}$ Christine R. Hamiel, BS, ${ }^{c}$ Richard Klaghofer, PhD, \\ Andreas Zollinger, MD, ${ }^{e}$ and Christoph K. Hofer, MD, DEAA ${ }^{\mathrm{e}}$
}

From the Institute of Anaesthesiology, ${ }^{\mathrm{a}}$ University Hospital Lausanne, Lausanne, Switzerland; the Department of Anesthesia and Perioperative Care, ${ }^{\mathrm{b}}$ University of California San Francisco, San Francisco, Calif; the Division of Cardiothoracic Surgery, ${ }^{\mathrm{c}}$ University of Colorado Health Sciences Centre, Denver, Colo; the Department of Psychosocial Medicine, ${ }^{\mathrm{d}}$ University Hospital Zurich, Zurich, Switzerland; and the Institute of Anaesthesiology and Intensive Care Medicine, ${ }^{\mathrm{e}}$ Triemli City Hospital Zurich, Zurich, Switzerland.

Material support was provided by Sienco Inc, Arvada, Colo.

Received for publication Oct 16, 2005; revisions received Dec 20, 2005; accepted for publication Jan 12, 2006.

Address for reprints: Christoph K. Hofer, $\mathrm{MD}$, Institute of Anaesthesiology and Intensive Care Medicine, Triemli City Hospital Zurich, Birmensdorferstrasse 497, CH-8063 Zurich, Switzerland (E-mail: Christoph.hofer@triemli.stzh.ch).

*Michael A. Tucci and Michael T. Ganter contributed equally to this work.

J Thorac Cardiovasc Surg 2006;131:1314-22 $0022-5223 / \$ 32.00$

Copyright $(\odot) 2006$ by The American Association for Thoracic Surgery

doi:10.1016/j.jtcvs.2006.01.041
Objective: Reliable platelet function monitoring is desirable in patients treated with glycoprotein IIb/IIIa receptor inhibitors. The aim of the present laboratory-based study was to assess platelet function after administration of clinically relevant doses of the glycoprotein IIb/IIIa antagonist tirofiban with or without heparin by using Sonoclot (Sienco Inc) and platelet aggregometry.

Methods: Tirofiban $\left(0-100 \mathrm{ng} \cdot \mathrm{mL}^{-1}\right)$ and heparin $\left(0\right.$ or $\left.1 \mathrm{U} \cdot \mathrm{mL}^{-1}\right)$ were added to blood samples obtained from 20 healthy volunteers. Coagulation analysis was performed on citrated whole blood by using the Sonoclot analyzer. The glass bead-activated test and the new glass bead test with heparinase were used. The results were compared with adenosine-5' -diphosphate-activated platelet aggregometry.

Results: Administration of tirofiban showed a similar increase of platelet inhibition detected with the Sonoclot glass bead-activated test and glass bead test with heparinase, as well as by means of aggregometry. Bias between the different techniques was comparable; Spearman rank correlation was strong (glass beadactivated test vs aggregometry: $\rho=0.823, P<.001$; glass bead test with heparinase vs aggregometry: $\rho=0.856, P<.001)$. After additional administration of heparin, platelet inhibition was only comparable for the glass bead test with heparinase and aggregometry, and the correlation coefficient remained unchanged for the glass bead test with heparinase versus aggregometry $(\rho=0.878, P<.001)$. By contrast, the glass bead-activated test showed a nearly complete platelet inhibition with a significant bias compared with the glass bead test with heparinase and aggregometry. Correlation was weak for the glass bead-activated test versus aggregometry $(\rho=0.407, P=.004)$.

Conclusions: When compared with platelet aggregometry, the glass bead-activated test from Sonoclot reliably detects glycoprotein IIb/IIIa receptor inhibition with tirofiban in unheparinized whole blood. However, in heparinized blood the glass bead test with heparinase is essential to accurately assess platelet function.

$\mathrm{P}$ harmacologic inhibition of platelet glycoprotein (GP) IIb/IIIa receptors is an established adjunct in the management of patients with acute coronary syndromes and in patients undergoing percutaneous coronary interventions. ${ }^{1}$ Tirofiban, an intravenously administered, short-acting, nonpeptide tyrosine derivative, acts as a reversible and selective antagonist of the GP IIb/IIIa receptor. After a 30-minute infusion of the recommended dose, platelet aggregation is inhibited more than $90 \%$, whereas platelet function $(\mathrm{PF})$ returns to near-baseline levels within 4 to 8 hours after discontinuing the infusion. Tirofiban is now increasingly used together with heparin because it has been shown to improve outcome in patients with acute coronary syndrome by preventing the occurrence of acute cardiac ischemic events. ${ }^{2,3}$ However, on requiring emer- 


$$
\begin{aligned}
& \text { Abbreviations and Acronyms } \\
& \begin{aligned}
\mathrm{ACT} & =\text { activated clotting time } \\
\mathrm{CPB} & =\text { cardiopulmonary bypass } \\
\mathrm{GB} & =\text { glass bead-activated test } \\
\mathrm{GP} & =\text { glycoprotein } \\
\text { hepGB } & =\text { glass bead-activated test containing } \\
& \text { heparinase } \\
\mathrm{PF} & =\text { platelet function } \\
\mathrm{PPP} & =\text { platelet-poor plasma } \\
\mathrm{PRP} & =\text { platelet-rich plasma }
\end{aligned}
\end{aligned}
$$

gency surgical revascularization or other diagnostic and therapeutic interventions, these patients might be at an increased risk for excessive bleeding. ${ }^{4-6}$ Some data indicate that the risk of perioperative bleeding can be limited by promptly stopping infusion of the GP IIb/IIIa inhibitors before surgical intervention and by means of perioperative platelet transfusion, ${ }^{7-9}$ but discontinuing these live-saving drugs might be harmful and should be avoided if possible.

Reliable bedside perioperative monitoring of both anticoagulant and PF is necessary to effectively manage the desired hemostasis suppression and its reversal. One of the major limitations of measuring PF has been that no reliable practical test exists. The historical gold standard, the bleeding time test, is a very crude measurement technique of PF. Bleeding time tests are poorly reproducible, invasive, insensitive, and time consuming. Turbidimetric platelet aggregometry, a clinical standard to measure PF ex vivo, requires specialized laboratory resources and is cumbersome to perform. ${ }^{10}$ A number of new testing methods have recently been developed to easily monitor PF at the patient's bedside. ${ }^{11}$ One of these devices, the Sonoclot Coagulation and Platelet Function Analyzer (Sienco Inc) provides pointof-care monitoring of the coagulation process from initial fibrin formation through platelet activation and clot retraction. ${ }^{12}$ The Sonoclot analyzer has been shown to accurately assess impaired PF. ${ }^{13}$ However, Sonoclot analyses with standard activators are heparin sensitive, and the presence of heparin might artificially modify the PF results. To overcome this problem, a new test containing glass beads and heparinase has been developed for the Sonoclot analyzer: glass beads are required for contact activation of the coagulation cascade, and heparinase is used to inactivate present heparin.

The aim of this laboratory study was to evaluate the standard glass bead-activated test (GB) and a new glass bead-activated test containing heparinase (hepGB) for the Sonoclot analyzer in assessing PF and to compare these tests with turbidimetric platelet aggregometry. Blood samples were analyzed after addition of clinically relevant doses of tirofiban and heparin in vitro.

\section{Methods}

With institutional approval and written informed consent, 20 healthy volunteers without hereditary or acquired coagulation disorders (including pharmacologically induced coagulopathies; ie, treatment with any anticoagulants or antiplatelet drugs 14 days before blood withdrawal) were included. Each volunteer donated $35 \mathrm{~mL}$ of blood, which was placed in citrated tubes (sodium citrate, $0.109 \mathrm{~mol} \cdot \mathrm{L}^{-}{ }^{1}$; BD Vacutainer, Becton Dickinson) by means of venipuncture. An initial Vacutainer tube was discarded before collection of the test samples. Baseline measurements included hemoglobin concentration, platelet count, prothrombin time, activated partial thromboplastin time, and celite-based activated clotting time (ACT).

Ten milliliters of citrated blood was used for whole blood coagulation measurements (ie, Sonoclot analyses). Another $20 \mathrm{~mL}$ of blood was processed for platelet aggregometry: the samples were centrifuged to obtain platelet-rich plasma (PRP; $135 g$ for 15 minutes) and platelet-poor plasma (PPP; $2000 \mathrm{~g}$ for 20 minutes). PPP was used to adjust the platelet count of PRP to within $10 \%$ of baseline platelet count.

Tirofiban (Merck \& Co, Inc) was added to the samples to obtain a final concentration of $0,12.5,25,50$, and 100 $\mathrm{ng} \cdot \mathrm{ml}^{-1}$. All samples were analyzed without and with porcine heparin (Liquemin, Roche Pharma) in a final concentration of 1 $\mathrm{U} \cdot \mathrm{ml}^{-1}$ (Figure 1).

\section{Sonoclot Analysis}

The Sonoclot analyzer is a point-of-care automated coagulation analyzer and has been described previously in detail. ${ }^{12,13}$ Briefly, the measurement is based on the detection of viscoelastic changes of a whole blood sample after activation with different coagulation activators (glass beads were used for this study). The Sonoclot analyzer provides information on the entire hemostasis process both in a qualitative graph, known as the Sonoclot signature (Figure 2, A), and as quantitative results: ACT (onset of clot formation), clot rate (maximum slope of the Sonoclot signature during initial fibrin polymerization), and $\mathrm{PF}$ (timing and quality of clot retraction). ${ }^{12} \mathrm{PF}$ is a calculated value, derived by using an automated numeric integration of changes in the Sonoclot signature after fibrin formation has completed (see manufacturer's reference). The nominal range of values for the PF goes from 0, representing no PF (no clot retraction and flat Sonoclot signature after fibrin formation), to approximately 5, representing strong PF (clot retraction occurs sooner and is very strong, with clearly defined, sharp peaks in the Sonoclot signature after fibrin formation). All Sonoclot analyses were performed at $37^{\circ} \mathrm{C}$ in duplicate by using standard GB (Sienco Inc) and hepGB (0.17 U of heparinase per test; Sienco Inc). Recalcification of the sample was done by adding $13 \mu \mathrm{L}$ of $0.25 \mathrm{~mol} \cdot \mathrm{L}^{-1} \mathrm{CaCl}_{2}$ to the test cuvette. Subsequently, $320 \mu \mathrm{L}$ of blood specimen was dispensed with a pipette, mixed, and analyzed. In the present study, we used 


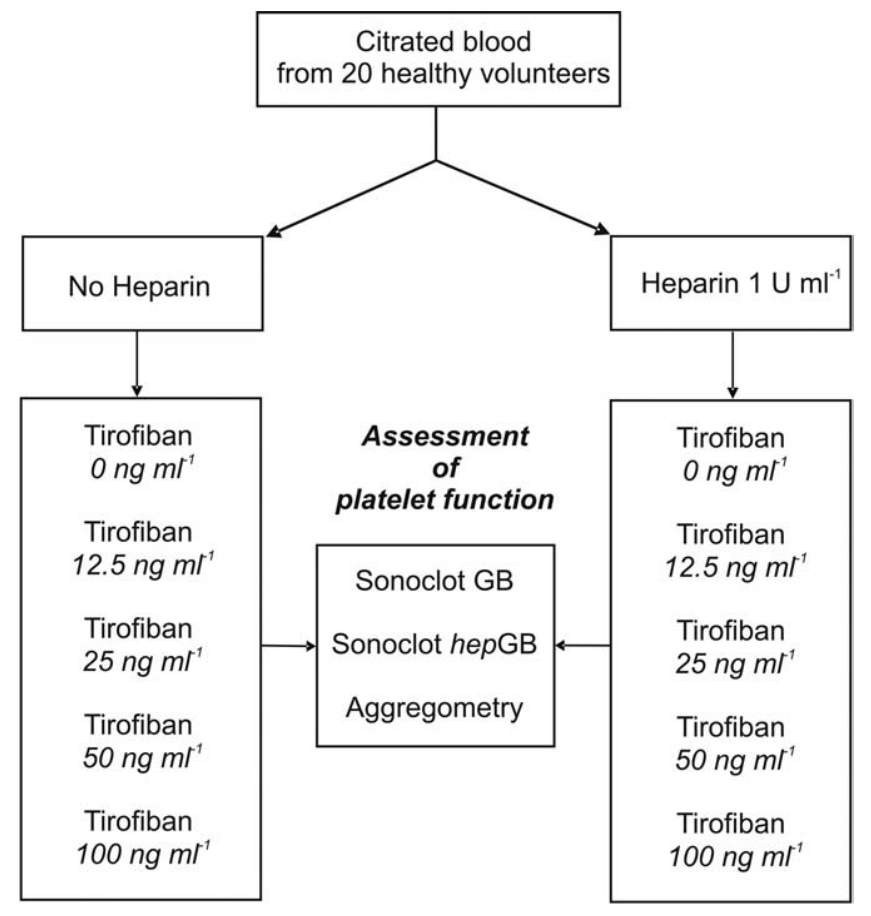

Figure 1. Study overview. Sonoclot GB, Glass bead-activated cuvettes; Sonoclot hepGB, glass bead-activated cuvettes with heparinase; Aggregometry, adenosine-5'-diphosphate-activated, turbidimetric platelet aggregometry.

Software Version 2.0.3 (release 04/2002) from the Sonoclot Analyzer.

\section{Platelet Aggregation}

Blood samples were centrifuged to obtain PRP and PPP, as described above. Platelet aggregation was optically monitored by using a 2-channel turbidimetric platelet aggregometer (Sienco Inc). Three hundred microliters of plasma was stirred in an aggregometer cuvette containing $20 \mu \mathrm{mol} \cdot \mathrm{L}^{-1}{ }^{1}$ adenosine-5' - diphosphate (Sigma Diagnostics). Measurements were performed at $37^{\circ} \mathrm{C}$ in duplicate, and PRP aggregation was measured against PPP aggregation. A baseline aggregation response (no tirofiban or heparin present) was determined for each donor. The percentage of aggregation was determined at the point of maximum light transmission obtained within 5 minutes.

\section{Data Analysis}

Statistical analysis was done with StatView for Windows version 5.01 (SAS Institute Inc) and SPSS for Windows Release 12.0.2 (SPSS Inc). The hypothesis of normal data distribution was rejected by using the Kolmogorov-Smirnov test. Effects of different concentrations of tirofiban on PF determined by means of the GB test, the hepGB test, and aggregometry with and without heparin were determined by using the Friedman test (repeated measurements). The Wilcoxon signedrank test was calculated to assess the effect of heparin on both Sonoclot tests and aggregometry. The percentage of platelet inhibition was calculated by using the following formulas:

\author{
$\%$ Platelet inhibition $_{\text {Sonoclot }}$ \\ $=($ Baseline platelet function \\ - Platelet function after tirofiban with or without heparin)/ \\ (Baseline platelet function) $\times 100$

$$
\begin{aligned}
\% \text { Platelet inhibition } & \text { Aggregometry } \\
= & (\% \text { Baseline aggregation } \\
& -\% \text { Aggregation after tirofiban with or without heparin }) / \\
& (\% \text { Baseline aggregation }) \times 100
\end{aligned}
$$

Bias between the percentage of platelet inhibition Sonoclot $_{\text {and }}$ the percentage of platelet inhibition Aggregometry was calculated as the difference of the percentage of platelet inhibition Sonoclot $_{\text {(GB }}$ and hepGB tests) and the percentage of platelet inhibition Aggregometry The Wilcoxon signed-rank test and Spearman rank correlation were used to compare the percentage of platelet inhibition assessed by the different testing methods. Test variability of duplicate measurements was calculated as a percentage of the mean percentage degree of platelet inhibition. Unless otherwise stated, nonparametric data are presented as median/interquartile range.

\section{Results}

Baseline coagulation parameters of the 20 volunteers (mean age, 36 [SD, 8] years; female/male ratio, 8/12) were within normal ranges, mean hemoglobin value was $14.0 \mathrm{~g} \cdot \mathrm{dL}-{ }^{1}$ $\left(\mathrm{SD}, 1.3 \mathrm{~g} \cdot \mathrm{dL}^{1}\right)$, and mean platelet count was $244 \times$ $10^{3} \cdot \mathrm{mm}^{-3}\left(\mathrm{SD}, 29 \times 10^{3} \cdot \mathrm{mm}^{-3}\right)$.

\section{Measurements Without Heparin}

Sonoclot signatures from one representative patient are shown in Figure 2, $B$, by using hepGB tests. The addition of tirofiban in increasing concentrations led to a similar, significant, and gradual decrease of PF assessed with the Sonoclot analyzer by using GB and hepGB tests, as well as by means of aggregometry (Table 1). Accordingly, the calculated percentage of platelet inhibition showed a significant stepwise increase for all 3 testing methods (Figure 3, A). The bias between the percentage of platelet inhibition assessed by using the Sonoclot analyzer (GB/hepGB tests) and the percentage of platelet inhibition measured by using aggregometry was not significant and decreased with increasing concentration of tirofiban (Figure 4, $A$ ). The bias of the percentage of platelet inhibition between the GB test and the hepGB test was $-0.3 / 14.4 \%$ (mean $[\mathrm{SD}]=-1.8$ [11.3\%]; $P=.162$ ). There was a significant and strong correlation of the percentage of platelet inhibition assessed by using the Sonoclot analyzer and aggregometry (Table 2).

\section{Measurements After Addition of Heparin}

After administration of heparin $\left(1 \mathrm{U} \cdot \mathrm{mL}^{-1}\right)$, mean baseline kaolin ACT increased from 118 (SD, 23) to 280 (SD, 75) seconds. PF analysis showed comparable patterns with the Sonoclot hepGB test and aggregometry for the 5 different tirofiban concentrations (Table 1 and Figure 3,B). Bias and 

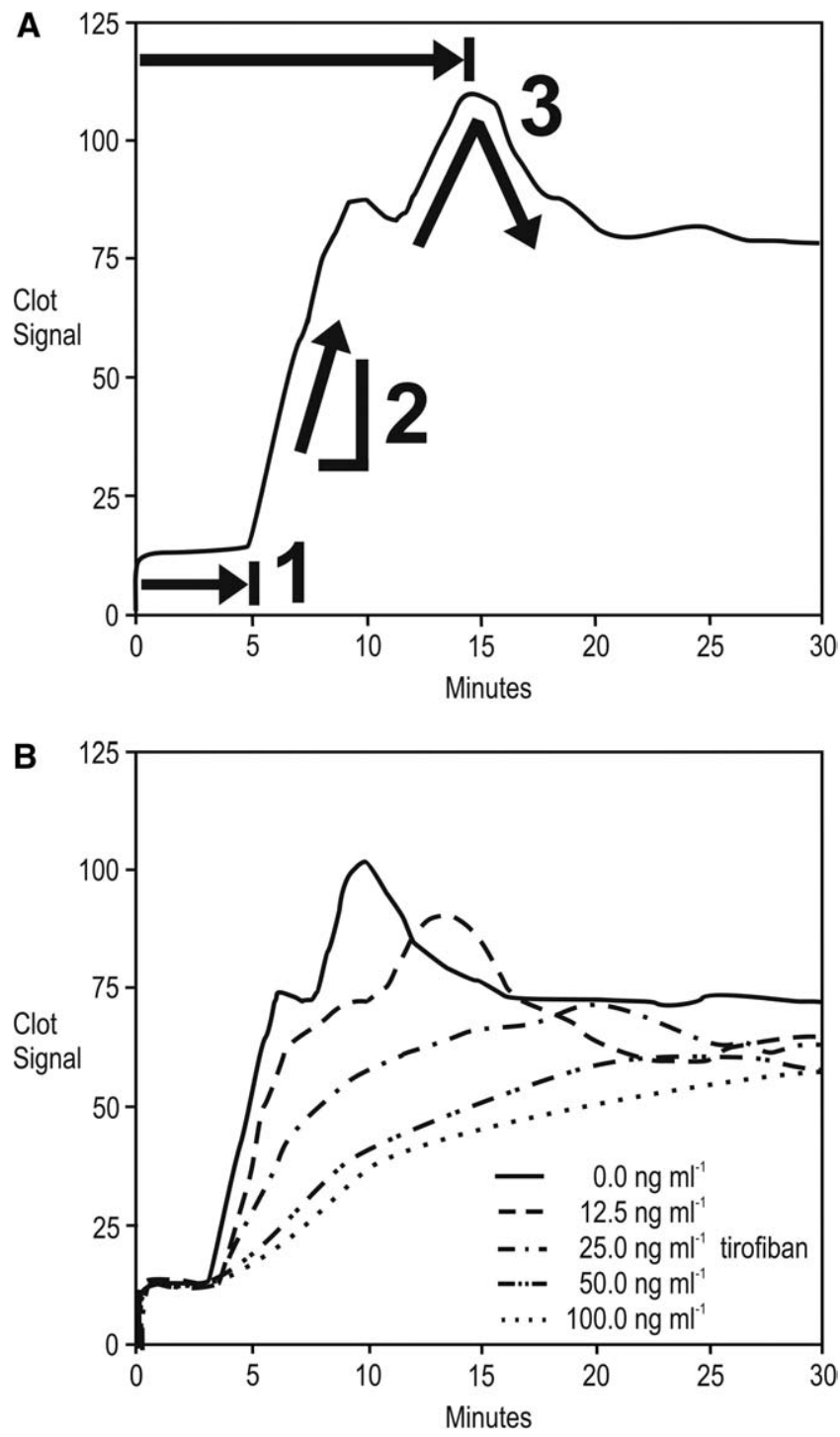

Figure 2. Sonoclot signatures. A, Typical Sonoclot signature. The following quantitative results are obtained: (1) activated clotting time (onset of clot formation); (2) clot rate (maximum slope of the Sonoclot signature during initial fibrin polymerization), and (3) platelet function (timing and quality of clot retraction). B, Sonoclot signatures from one representative patient after administration of different concentrations of tirofiban to blood samples in vitro (final concentration $=0,12.5,25,50$, and $100 \mathrm{ng} \cdot \mathrm{ml}^{-1}$ ).

correlation for the percentage of platelet inhibition was comparable with the values obtained for measurements without heparin (Figure 4, B, and Table 2). By contrast, assessment with the Sonoclot GB test without heparinase showed a nearly complete loss of PF after addition of tirofiban. A significant bias of the percentage of platelet inhibition assessed with the Sonoclot GB test and aggregometry for all tirofiban concentrations was observed (Figure 4, B). Bias of the percentage of plat elet inhibition between the GB and hepGB tests was 20.3/ $28.0 \%$ (mean $[\mathrm{SD}]=26.3[22.1 \%] ; P<.001)$. Correlations of the percentage of platelet inhibition comparing the GB with the hepGB test, as well as the GB test with aggregometry, were weak (Table 2).

\section{Test Variability}

In measurements without heparin, test variability (related to the percentage of platelet inhibition) for the GB test was $6.6 / 8.4 \%$ (mean $[\mathrm{SD}]=9.8[9.5 \%]$ ), for the hepGB test was $6.0 / 8.9 \%$ (mean $[\mathrm{SD}]=8.3[7.9 \%]$ ), and for aggregometry was $6.4 / 7.3 \%$ (mean $[\mathrm{SD}]=6.7[5.3 \%]$ ). The addition of heparin induced a significant change of test variability for the GB test $(0.0 / 3.8 \%$; mean $[\mathrm{SD}]=10.1$ [27.9\%]; $P<$ $.001)$, whereas test variability for the hepGB test and aggregometry remained unchanged (hepGB test $=6.1 / 78.0 \%$; mean $[\mathrm{SD}]=8.4[8.5 \%] ; P=.717 ;$ aggregometry $=$ $6.0 / 9.1 \%$; mean $[\mathrm{SD}]=7.4[6.4 \%] ; P=.197)$.

\section{Discussion}

In the present in vitro study, platelet inhibition with tirofiban was reliably monitored with the hepGB test in both unheparinized and heparinized blood samples. The results with this hepGB test correlated well with those obtained with adenosine-5' -diphosphate-activated platelet aggregometry. By contrast, the GB test was only reliable to monitor platelet inhibition by tirofiban in unheparinized blood samples.

Shorter-acting platelet GP IIb/IIIa inhibitors like tirofiban have become an increasingly important therapeutic option in unstable coronary artery disease, as well as during and after percutaneous coronary interventions. ${ }^{14}$ In patients with acute coronary syndrome, treatment with tirofiban combined with heparin has been shown to improve patients outcome. ${ }^{2,3}$ However, platelet inhibition with GP IIb/IIIa inhibitors is not restricted to cardiac patients only. These drugs are also used in other clinical situations in which prevention of an arterial thrombosis is a primary goal. For example, GP IIb/IIIa antagonists have been successfully used as an adjunct in thrombolysis management of peripheral arterial occlusions. ${ }^{15}$ Because GP IIb/IIIa inhibitors impair PF considerably, one might expect that excessive bleeding during surgical intervention or major interventions might occur if timing and dosing are not adequate. Indeed, some studies with the long-acting GP IIb/IIIa inhibitor abciximab showed increased bleeding and transfusion requirements if surgical intervention had been performed within 12 hours of receiving this drug. ${ }^{16}$ However, the situation with the short-acting inhibitors tirofiban and eptifibatide is less clear. There are only a few case reports describing excessive intraoperative and postoperative hemorrhage after treatment with tirofiban or eptifibatide. ${ }^{4,5}$ Most studies showed either comparable or even reduced bleeding rates in patients undergoing cardiopulmonary bypass (CPB) 
TABLE 1. Platelet function assessed with Sonoclot GB/hepGB cuvettes and platelet aggregometry

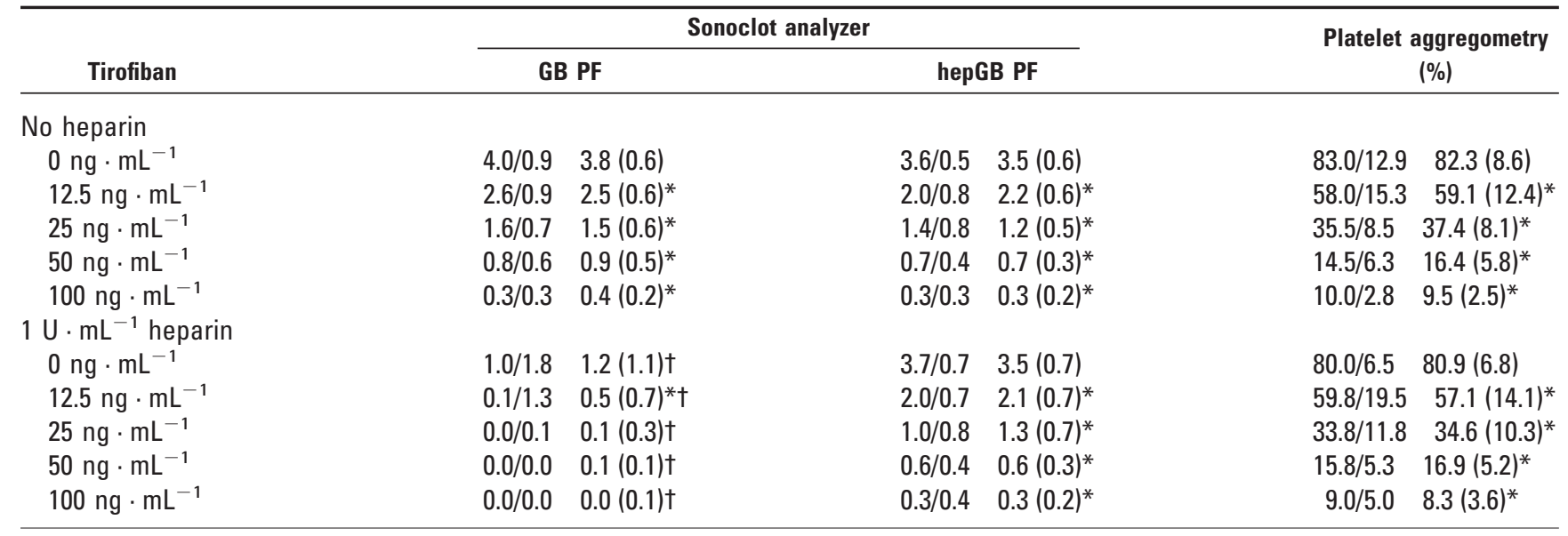

Data are presented as median/interquartile range and mean (SD). GB, Glass bead-activated test; hepGB, glass bead-activated test with heparinase; $P F$, platelet function; Aggregometry, adenosine-5'-diphosphate-activated, turbidimetric platelet aggregometry. $* P<.05$ for comparison of consecutive tirofiban concentrations, $\dagger P<.05$ for comparison of no heparin versus $1 \mathrm{U} \cdot \mathrm{mL}^{-1}$ heparin.

surgery. ${ }^{7,9}$ The question remains of whether short-acting GP $\mathrm{IIb} / \mathrm{III}$ inhibitors can inhibit platelet reactivity during $\mathrm{CPB}$, preventing platelet aggregation and adhesion and thus preserving platelet number and function after CPB ends. According to this theory, the key requirement would be that the $\mathrm{PF}$ is completely inhibited before heparinization and that this inhibition is quickly reversed after ending the $\mathrm{CPB}$, allowing adequate hemostasis (also called "platelet anesthesia"). ${ }^{17,18}$

Tirofiban is a reversible antagonist of fibrinogen binding to the GP IIb/IIIa receptor, the major platelet surface receptor involved in platelet aggregation. When administered intravenously, tirofiban inhibits platelet aggregation in a dose- and concentration-dependent manner. When administered according to the recommended regimen, more than $90 \%$ platelet inhibition is attained by the end of a 30-minute tirofiban infusion. It has a half-life of approximately 2 hours, and it is cleared from the plasma largely by means of renal excretion. In patients with atherosclerotic disease and impaired renal function, plasma clearance is significantly lower. Pharmacokinetics are fairly predictable in healthy patients, but this might not be the case in the typical patient receiving tirofiban. Furthermore, empiric dosing of GP IIb/ IIIa antagonists on a body weight or surface area basis might be suboptimal because of known interpatient variability ${ }^{19-21}$ based on variations of platelet count and the ability of these platelets to aggregate. The goal of PF monitoring during treatment with GP IIb/IIIa antagonists is to confirm adequate platelet inhibition and to possibly titrate the dose according to individual needs. On the other side, PF monitoring should allow detection of unwanted platelet inhibition, such as prolonged action after discontinuing these drugs in renal disease or CPB-related platelet dysfunctions. Normal platelet count and preserved $\mathrm{PF}$ are required for adequate perioperative hemostasis. In patients with acute coronary syndrome undergoing emergency cardiovascular surgery or other major interventions, GP IIb/IIIa inhibitor treatment is usually continued up until shortly before the procedure. Reliable bedside PF monitoring is highly desirable for optimal coagulation management to prevent high blood loss, which is related to impaired clinical outcomes, ${ }^{6}$ and might result in better clinical outcome. ${ }^{19}$

Traditional assays, such as turbidimetric platelet aggregometry, are still considered clinical standards of PF testing. Turbidimetric platelet aggregometry is one of the most widely used tests to identify and diagnose PF defects. However, conventional platelet aggregometry is labor intensive, costly, time consuming, and requires a high degree of experience and expertise to perform and interpret. Another important limitation of this technique is that platelets are tested under relatively low shear conditions and in free solution within PRP, conditions that do not accurately simulate primary hemostasis. ${ }^{11}$ Because of these disadvantages of conventional platelet aggregometry, new automated technologies have been developed to measure PF, as described previously in detail. ${ }^{10,11,22}$ Several techniques can be used at the bedside, such as ICHOR/Plateletworks (Helena Laboratories; comparing platelet counts in whole blood within a baseline EDTA tube and after activation/aggregation with a platelet agonist); PFA-100 (Dade Behring Inc; platelet adhesion/aggregation measured under high shear conditions in the presence of platelet activators), IMPACT (DiaMed AG; platelet adhesion/aggregation on a plate under applied shear stress in whole blood), Ultegra-RPFA (Accumetrics Inc; modified turbidimetric platelet aggregometry in whole blood), Mulitplate (Dynabyte $\mathrm{GmbH}$; impedance platelet aggregometry in whole blood), hemoStatus (Medtronic Inc; $\mathrm{PF}$ derived from measurement of the ACT in the presence or absence of platelet-activating factor), Hemostasis Analysis 

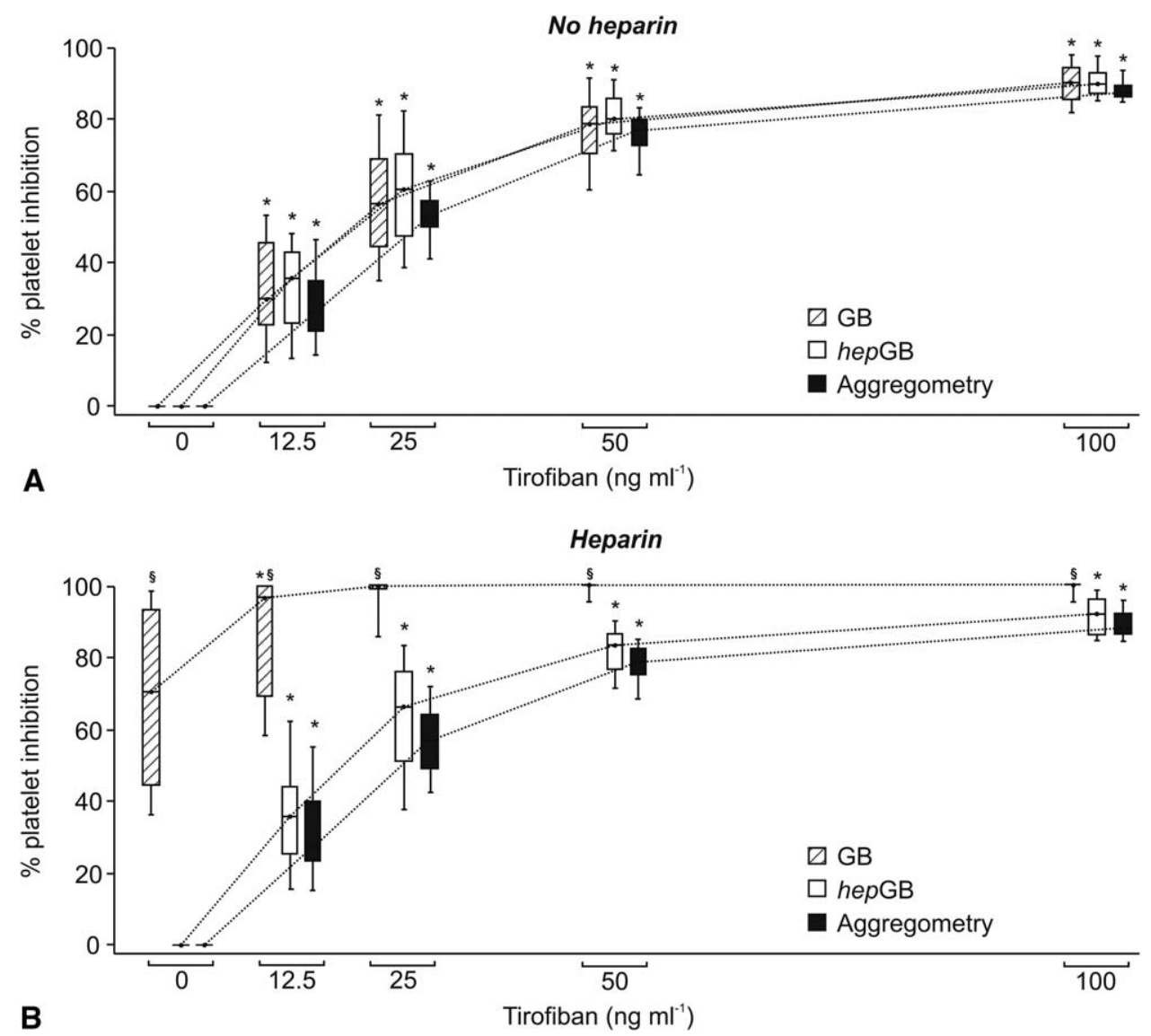

Figure 3. Percentage of platelet inhibition assessed by using the Sonoclot analyzer and platelet aggregometry $(A$, no heparin; B, $1 \mathrm{U} \cdot \mathrm{mL}^{-1}$ heparin). GB, Sonoclot glass bead-activated cuvettes; hepGB, Sonoclot glass beadactivated cuvettes with heparinase; Aggregometry, adenosine-5'-diphosphate-activated, turbidimetric platelet aggregometry. ${ }^{*} \boldsymbol{P}<.05$ for comparison of consecutive tirofiban concentrations, $\$ P<.05$ for comparisons of no heparin versus $1 \mathrm{U} \cdot \mathrm{mL}^{-1}$ heparin.

System (Hemodyne Inc; global hemostasis assessment in whole blood, PF derived from the force generated by platelets during clot retraction), thrombelastography/-metry (TEG 5000, Haemoscope Corp/ROTEM, Pentapharm GmbH; global hemostasis assessment in whole blood, PF derived from maximal clot-shear elasticity), or the Sonoclot Coagulation and Platelet Function Analyzer.

In contrast to most other PF monitoring devices, the Sonoclot analyzer provides bedside information on the entire hemostasis process, including PF, and has been shown to accurately assess impaired PF. ${ }^{13}$ In a clinical setting inhibition of platelet aggregation with GP IIb/IIIa antagonists is usually combined with heparin to inhibit thrombin generation. Combining these 2 drugs has a summation effect on the coagulation inhibition. The presence of heparin will mask PF information by preventing platelet activation. Therefore displayed PF results for heparinized blood samples treated with antiplatelet drugs might be unreliable unless heparinase is present. Our data with the Sonoclot analyzer show that detection of PF inhibition with the conventional GB test was only accurate in unheparinized blood samples after treatment with tirofiban. However, the new test containing glass beads and heparinase (hepGB) performed well in both unheparinized and heparinized blood samples. Heparinase enzymatically inactivates heparin in the sample so that PF can be adequately assessed. The hepGB test is designed for blood samples with therapeutic heparin levels $\left(0.6 \pm 0.3 \mathrm{U} \cdot \mathrm{mL}^{-1}\right)$ because the amount of heparinase in the test tube is able to immediately neutralize at least $1 \mathrm{U} \cdot \mathrm{mL}^{-1}$ of heparin.

The Sonoclot analyzer has been criticized because its results were influenced by age, sex, and platelet count. ${ }^{23}$ Additionally, studies showed poor reproducibility of some of the measured parameters, especially clot rate and $\mathrm{PF}^{22,24}$ However, others found the Sonoclot analyzer to be valuable and reliable in patients undergoing cardiac surgical procedures, ${ }^{13,25}$ and the 

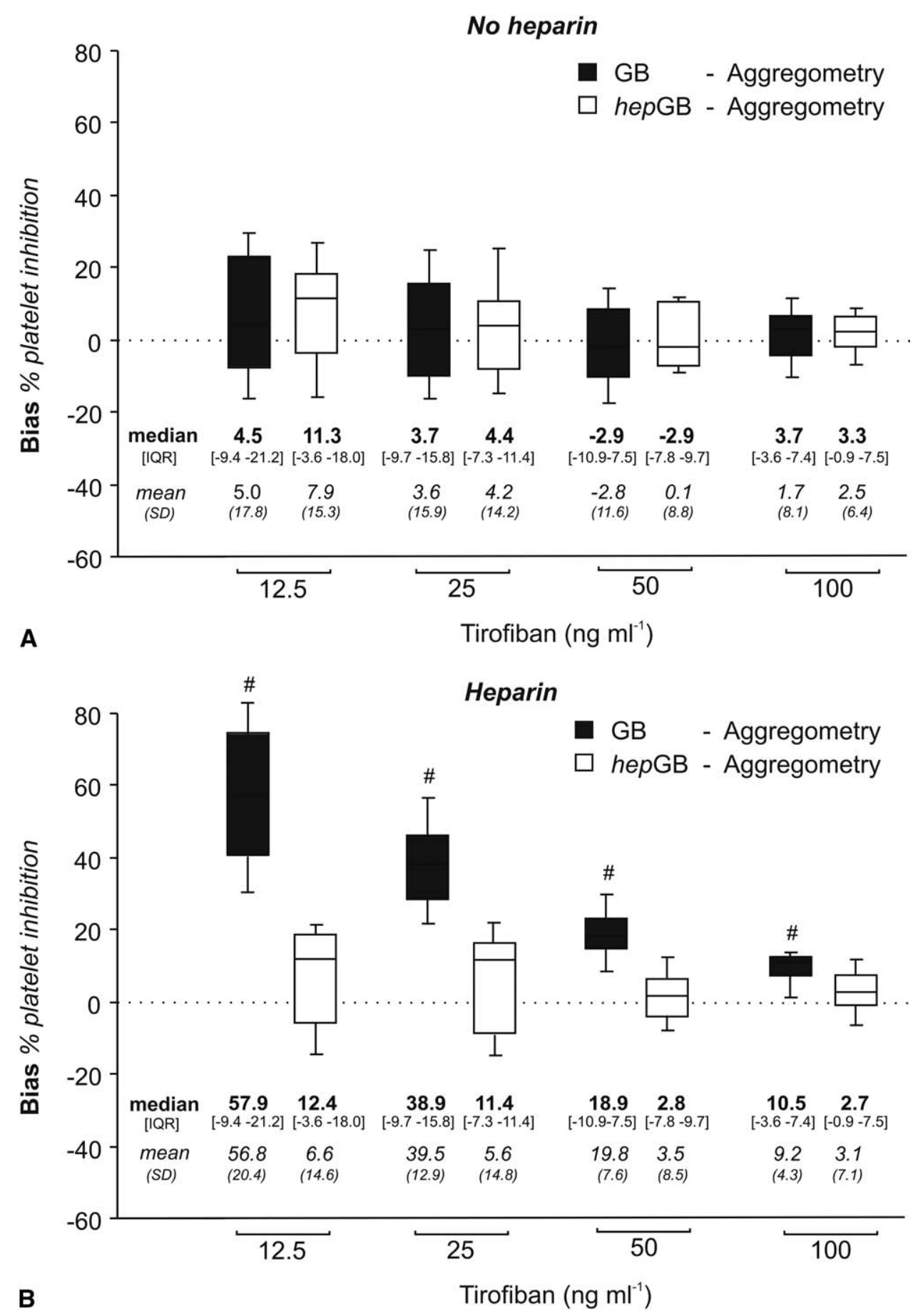

Figure 4. Bias of the percentage of platelet inhibition assessed by using the Sonoclot analyzer and platelet aggregometry (A, no heparin; B, $1 \mathrm{U} \cdot \mathrm{mL}^{-1}$ heparin). GB, Sonoclot glass bead-activated cuvettes; hepGB, Sonoclot glass bead-activated cuvettes with heparinase; Aggregometry, adenosine-5'-diphosphate-activated, turbidimetric platelet aggregometry; IQR, interquartile range; $S D$, standard deviation. Bias is defined as the percentage of platelet inhibition assessed with the Sonoclot analyzer minus the percentage of platelet inhibition measured by means of platelet aggregometry. $\# P<.05$ for comparison of the percentage of platelet inhibition assessed with the Sonoclot analyzer and the percentage of platelet inhibition measured by means of platelet aggregometry. 
TABLE 2. Spearman rank correlation coefficients comparing the percentage of platelet inhibition assessed with Sonoclot GB and hepGB cuvettes and platelet aggregometry

\begin{tabular}{lcc}
\hline $\begin{array}{l}\text { \% Platelet } \\
\text { inhibition }\end{array}$ & hepGB & Aggregometry \\
\hline $\begin{array}{l}\text { No heparin } \\
\text { GB }\end{array}$ & $0.892, P<.001$ & $0.823, P<.001$ \\
hepGB & - & $0.856, P<.001$ \\
$1 \mathrm{U} \cdot \mathrm{mL}^{-1}$ & & \\
GB & $0.385, P=.008$ & $0.407, P=.005$ \\
hepGB & - & $0.878, P<.001$ \\
\hline
\end{tabular}

$G B$, Glass bead-activated cuvettes; hepGB, glass bead-activated cuvettes with heparinase; Aggregometry, adenosine- $5^{\prime}$-diphosphate-activated, turbidimetric platelet aggregometry.

Sonoclot analyzer has even demonstrated a precision close to that of thrombelastography. ${ }^{26}$ In the present study we were able to show reliable results with reproducibility comparable with that of turbidimetric platelet aggregometry. One possible explanation for this better performance might be the different tests used in our study (GB and hepGB) compared with those used in earlier publications (predominantly testing of celiteactivated blood samples).

The present study has some limitations. An in vitro study does not necessarily reflect coagulation changes in vivo, and the clinical relevance of impaired PF cannot be shown (eg, bleeding of the patient or the need for platelet transfusion). The present study was performed with blood samples of healthy volunteers and administration of one GP IIb/IIIa antagonist (ie, tirofiban). Care should be taken to extrapolate these in vitro results to the in vivo surgical situation, as well as the results obtained with tirofiban to other GP IIb/IIIa antagonists. Further studies have to confirm our in vitro findings by using specimens obtained from patients who are receiving these agents as part of their clinical management. Additionally, we compared results from the Sonoclot analyzer (whole blood samples) with results from platelet aggregometry obtained in PRP. Coagulation processes in PRP might not accurately reflect primary hemostasis in whole blood. However, platelet aggregometry in PRP is the clinical standard to assess PF and measures the percentage of platelets that aggregate on stimulation with a platelet agonist. The Sonoclot analyzer does not measure PF directly but calculates PF from the quality of clot retraction. Furthermore, to compare results from the 2 measurement techniques, we calculated the percentage of platelet inhibition. This cannot be calculated in most patients because no baseline PF exists. In clinical practice the value given from the Sonoclot analyzer for PF gives an estimate of the current status, and change in PF over time can be monitored.

In conclusion, our in vitro data indicate that GP IIb/IIIa inhibition with tirofiban is accurately reflected by the Sonoclot analyzer in the presence of heparin by using a heparinase- containing glass bead test (hepGB test). The standard glass bead test (GB test), however, is only reliable in assessing PF in situations in which no heparin is used.

\section{References}

1. Neumann FJ, Hochholzer W, Pogatsa-Murray G, Schomig A, Gawaz M. Antiplatelet effects of abciximab, tirofiban and eptifibatide in patients undergoing coronary stenting. J Am Coll Cardiol. 2001;37:1323-8.

2. Inhibition of the platelet glycoprotein IIb/IIIa receptor with tirofiban in unstable angina and non-Q-wave myocardial infarction. Platelet Receptor Inhibition in Ischemic Syndrome Management in Patients Limited by Unstable Signs and Symptoms (PRISM-PLUS) Study Investigators. N Engl J Med. 1998;338:1488-97.

3. Effects of platelet glycoprotein IIb/IIIa blockade with tirofiban on adverse cardiac events in patients with unstable angina or acute myocardial infarction undergoing coronary angioplasty. The RESTORE Investigators. Randomized Efficacy Study of Tirofiban for Outcomes and REstenosis. Circulation. 1997;96:1445-53.

4. Brose S, Sirbu H, Engel M, Kuhlen R, Autschbach R. Successful use of recombinant Factor VIIa in a patient with intractable bleeding during extracorporeal membrane oxygenation. Thorac Cardiovasc Surg. 2005;53:389-90.

5. Waters JH, Anthony DG, Gottlieb A, Sprung J. Bleeding in a patient receiving platelet aggregation inhibitors. Anesth Analg. 2001;93:878-82.

6. Brown DL. Deaths associated with platelet glycoprotein IIb/IIIa inhibitor treatment. Heart. 2003;89:535-7.

7. Bizzarri F, Scolletta S, Tucci E, Lucidi M, Davoli G, Toscano T, et al. Perioperative use of tirofiban hydrochloride (Aggrastat) does not increase surgical bleeding after emergency or urgent coronary artery bypass grafting. J Thorac Cardiovasc Surg. 2001;122:1181-5.

8. Pang JT, Fort S, Della SA, Cohen EA. Emergency coronary artery bypass surgery in the era of glycoprotein $\mathrm{IIb} / \mathrm{III}$ a receptor antagonist use. J Card Surg. 2002;17:425-31.

9. Genoni M, Zeller D, Bertel O, Maloigne M, Turina M. Tirofiban therapy does not increase the risk of hemorrhage after emergency coronary surgery. J Thorac Cardiovasc Surg. 2001;122:630-2.

10. Rand ML, Leung R, Packham MA. Platelet function assays. Transfus Apheresis Sci. 2003;28:307-17.

11. Harrison P. Platelet function analysis. Blood Rev. 2005;19:111-23.

12. Hett DA, Walker D, Pilkington SN, Smith DC. Sonoclot analysis. Br J Anaesth. 1995;75:771-6.

13. Miyashita T, Kuro M. Evaluation of platelet function by Sonoclot analysis compared with other hemostatic variables in cardiac surgery. Anesth Analg. 1998;87:1228-33.

14. Anne G, Gruberg L. Platelet glycoprotein IIb/IIIa inhibitors during percutaneous coronary interventions: a pharmacological and clinical review. Exp Opin Pharmacother. 2004;5:335-48.

15. Ouriel K. Use of concomitant glycoprotein IIb/IIIa inhibitors with catheter-directed peripheral arterial thrombolysis. J Vasc Interv Radiol. 2004;15:543-6.

16. Gammie JS, Zenati M, Kormos RL, Hattler BG, Wei LM, Pellegrini $\mathrm{RV}$, et al. Abciximab and excessive bleeding in patients undergoing emergency cardiac operations. Ann Thorac Surg. 1998;65:465-9.

17. Suzuki Y, Hillyer P, Miyamoto S, Niewiarowski S, Sun L, Rao AK, et al. Integrilin prevents prolonged bleeding times after cardiopulmonary bypass. Ann Thorac Surg. 1998;66:373-81.

18. Hiramatsu Y, Gikakis N, Anderson HL III, Gorman JH III, Marcinkiewicz C, Gould RJ, et al. Tirofiban provides "platelet anesthesia" during cardiopulmonary bypass in baboons. J Thorac Cardiovasc Surg. 1997;113:182-93.

19. Kereiakes DJ, Broderick TM, Roth EM, Whang D, Shimshak T, Runyon JP, et al. Time course, magnitude, and consistency of platelet inhibition by abciximab, tirofiban, or eptifibatide in patients with unstable angina pectoris undergoing percutaneous coronary intervention. Am J Cardiol. 1999;84:391-5.

20. Steinhubl SR, Kottke-Marchant K, Moliterno DJ, Rosenthal ML, Godfrey NK, Coller BS, et al. Attainment and maintenance of platelet inhibition through standard dosing of abciximab in diabetic and nondiabetic patients undergoing percutaneous coronary intervention. Circulation. 1999;100:1977-82. 
21. Wheeler GL, Braden GA, Bray PF, Marciniak SJ, Mascelli MA, Sane DC. Reduced inhibition by abciximab in platelets with the PlA2 polymorphism. Am Heart J. 2002;143:76-82.

22. McKenzie ME, Gurbel PA, Levine DJ, Serebruany VL. Clinical utility of available methods for determining platelet function. Cardiology. 1999;92:240-7.

23. Horlocker TT, Schroeder DR. Effect of age, gender, and platelet count on Sonoclot coagulation analysis in patients undergoing orthopedic operations. Mayo Clin Proc. 1997;72:214-9.
24. Ekback G, Carlsson O, Schott U. Sonoclot coagulation analysis: a study of test variability. J Cardiothorac Vasc Anesth. 1999;13:393-7.

25. Saleem A, Blifeld C, Saleh SA, Yawn DH, Mace ML, Schwartz M, et al. Viscoelastic measurement of clot formation: a new test of platelet function. Ann Clin Lab Sci. 1983;13:115-24.

26. Forestier F, Belisle S, Contant C, Harel F, Janvier G, Hardy JF. [Reproducibility and interchangeability of the Thromboelastograph, Sonoclot and Hemochron activated coagulation time in cardiac surgery]. Can J Anaesth. 2001;48:902-10. 